\title{
Performance Evaluation of Single path and Multipath regarding Bandwidth and Delay
}

\author{
Maryada \\ M.Tech. Student, Department \\ of Computer Science \& \\ Applications, Kurukshetra \\ University, Kurukshetra- \\ 136119
}

\author{
Shalini Aggarwal \\ Teacher Fellow, Department of \\ Computer Science \& \\ Applications, Kurukshetra \\ University, Kurukshetra- \\ 136119
}

\author{
Pardeep Mittal, PhD \\ Assistant Professor, \\ Department of Computer \\ Science \& Applications, \\ Kurukshetra University, \\ Kurukshetra-136119
}

\begin{abstract}
This paper is all about finding multipaths available in the network using disjoint edges and disjoint nodes strategy. In single path routing congestion is a big issue, multipath routing is helpful to find less-congested or congestion free route from source to destination. Disjoint nodes means having no repetition of node that are followed earlier by the data packets to avoid packet congestion or load over the network. Disjoint edge means having no repetition of edges while following the path. The paths have been choosen on the basis of available bandwidth, time required, sequential order of node or hopcount. The sequence of every available single path from the given source to destination have been found using minimum hop dijkstra algorithm so that it is shortest among all available remaining paths. Results are shown on the basis of bandwidth and delay for single path and multipath routing (using disjoint node and disjoint edges).
\end{abstract}

\section{Keywords}

Disjoint edges, Disjoint nodes, Multipath Routing, Recovery Path Failure, and Single Path Routing.

\section{INTRODUCTION}

Routing is finding best path among all available paths. Nowa-days a lot of data is to be transmitted from one place to another. For this transmission of data single path and multipath routing schemes can be used. In single path only one route is used for transmission of data from source to destination. It is destination based load intensive technique. Shortest path algorithms can be divided into two categories link state routing and distance vector routing. Dijkstra algorithm [1] is one of the most famous algorithm for finding shortest path that comes under Link State Routing and is used in Repeated manner. Bellman Ford algorithm [2] may also use for shortest path calculation that comes under Distance Vector Routing. Single path routing is easy to implement and handle, but when data size increases various problems such as congestion, buffering, packet loss, power consumption, bottleneck problem, network failure may occur. Therefore multipath routing becomes a necessity in such cases.

In Multipath routing we have more than one path are available from source to destination. In case of network failure we have backup recovery paths that reduces delay time and congestion problem. Multipath routing maintains routing tables containing information regarding bandwidth, no of hopes, source, destination, cost, load, delay etc. Multipath networks are fault tolerant as bandwidth increases throughput also increases.

A number of algorithms exists for multipath routing such as ECMP [3] [4], DLFA, MRC, Popularity Aware Load
Balancing PALB [5] etc. ECMP can be combined with any of techniques to improve its results. To avoid congestion we have different strategies two of them are disjoint edge and disjoint node.

\section{ALGORITHMS USED IN SINGLE AND MULTIPATH ROUTING IN PRESENT WORK [] \\ 2.1 Single Shortest Path Algorithm (Dijkstra) \\ 1 Initialization: \\ $2 \mathrm{~S}=\{\mathrm{u}\}$ \\ 3 for all nodes $\mathrm{v}$ \\ 4 if $\mathrm{v}$ adjacent to $\mathrm{u}\{$ \\ $5 \quad \mathrm{D}(\mathrm{v})=\mathrm{c}(\mathrm{u}, \mathrm{v})$ \\ 6 else $\mathrm{D}(\mathrm{v})=\infty$ \\ 7 Loop \\ 8 find $w$ not in $\mathrm{S}$ with the smallest $\mathrm{D}(\mathrm{w})$ \\ 9 add $w$ to $S$ \\ 10 update $\mathrm{D}(\mathrm{v})$ for all $\mathrm{v}$ adjacent to $\mathrm{w}$ and not in $\mathrm{S}$ : \\ $11 \quad \mathrm{D}(\mathrm{v})=\min \{\mathrm{D}(\mathrm{v}), \mathrm{D}(\mathrm{w})+\mathrm{c}(\mathrm{w}, \mathrm{v})\}$}

12 until all nodes in $\mathrm{S}$

\subsection{Edge-Disjoint and Node disjoint Shortest Pair Algorithm}

1. Calculate the shortest path from current edge/node to all neighboring edges/nodes using Dijkstra algorithm.

2. Replace current path with the smallest one calculated among all paths.

3. Now second shortest path is calculated for modified network and we need a modified Dijkstra's Algorithm that can handle loop-free directed negative edges.

4. Change the edges/nodes to original network.

5. Delete those edges/nodes that are common to both found shortest paths and make a new group for unused edges and nodes.

\section{RELATED WORK}

(S.Nelakuditi and Z.L.Zhang, 2001) [7] Discussed that congestion can be minimized by diverting traffic over 
different paths and we should keep in mind two things i.e. total no of paths required and criteria to select path. Discussion over ECMP, OSPF-OMP, Wide Disjoint Paths, EBP (Equalizing Blocking Probability) its objective is to find proportion of set. The paper proposed hybrid approach is having better results than that of WSP.

(Yong Oh Lee et al, 2010) [] Paper is about proactive fast failure recovery mechanism. The paper explained that extra infrastructure can be exploited for building different disjoint paths length equal to the original path length, it minimizes the infrastructure cost in multipath environment. Many recovery techniques are available such as MRC (Multiple Routing Configuration), Not- via, LFA (Loop Free Alternatives). The authors proposed disjoint MRC, DLFA, DNot-via that are helpful to find disjoint paths.

(T.Janaszka et al, 2011) [9] In this different load balancing techniques are discussed that effect network efficiency. They proposed an approach called as PALB (Popularity Aware Load Balancing). The paper provided an overview about ECMP, Content Centric Networks and Content Decentralized networks. The authors proposed concept of caching of multipath routing and Link-level load balancing due to which network delay can be minimized. It increases the efficiency of the network by filtering popular content.

(N.Krishna Chaitanya et. al, 2015) [10] From this paper we a comparative view over different schemes is available for load distribution in multipath routing. Comparison is done on the basis of two techniques first is hope by hope and second is source routing. Source routing is more famous than previous one. Their comparative study is very helpful to implement various algorithms/ methods to multipath routing.

(F.Iqbal et all, 2015) [11] Authors provided an over view for Single path, multipath, disjoint path with example and their complexity. For disjoint path calculation Iterative Disjoint Path (IDP) algorithm is used but it fails sometimes to give real disjoint paths and enters into trap topology, Bhandari algorithm is used to overcome this problem.

\section{EXAMPLE NETWORK}

\subsection{Tool used: Matlab}

Matlab is abbreviation for Matrix Laboratory it is developed by MathWorks mainly designed for engineering and scientific purpose. It is a well-known worldwide used programming environment for visualization, communication, Algorithm development, image processing and for mathematical calculations. It is directed input graph with 7 edges where node 1 is source and node 4 is destination.

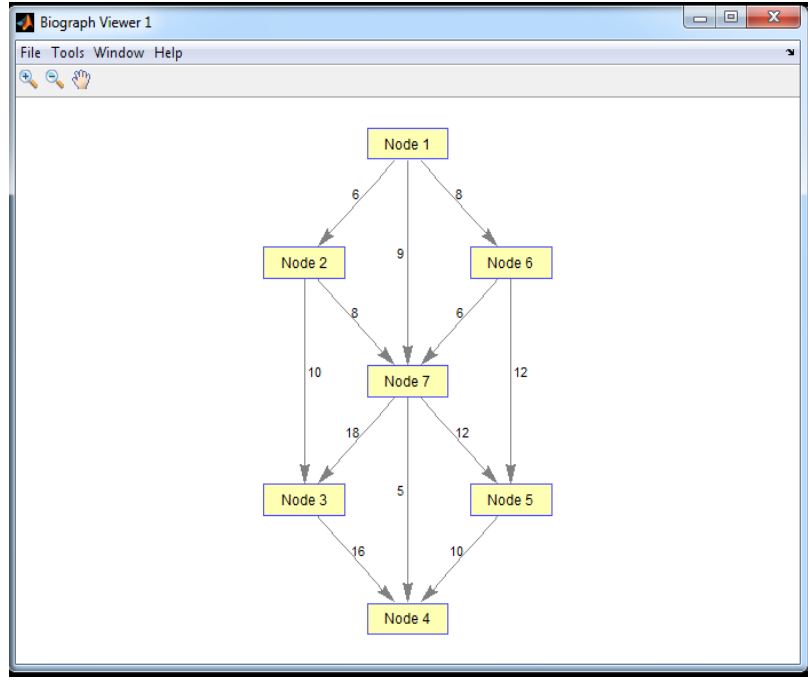

Figure 1 with 7 vertices and 12 edges

According to graph table 1 is calculated. Table contains Edges, Bandwidth and Delay on the basis of which we are calculating performance of the network. From the table we will get two graphs named figure 2 where received bandwidth is shown for single path and multipath (disjoint edge, disjoint node) figure 3 where success rate is shown for single path and multipath (disjoint node, disjoint edge).

In figure 2 single path routing bandwidth received is less than multipath routing due to which congestion occurs and network performance degrades. Results for disjoint edge and disjoint node are better than single path routing in our graph.

Table 1 for input graph

\begin{tabular}{|l|l|l|l|}
\hline Serial no. & Edges & Bandwidth & Delay \\
\hline 1 & $1-2$ & 6 & 3 \\
\hline 2 & $1-6$ & 8 & 4 \\
\hline 3 & $1-7$ & 9 & 5 \\
\hline 4 & $2-3$ & 10 & 5 \\
\hline 5 & $2-7$ & 8 & 3 \\
\hline 6 & $3-4$ & 16 & 6 \\
\hline 7 & $5-4$ & 10 & 2 \\
\hline 8 & $6-5$ & 12 & 9 \\
\hline 9 & $6-7$ & 6 & 4 \\
\hline 10 & $7-3$ & 18 & 3 \\
\hline 11 & $7-4$ & 5 & 5 \\
\hline 12 & $7-5$ & 12 & 2 \\
\hline
\end{tabular}

In figure 3 success rates for packets in single path routing is less than that of multipath routing. 


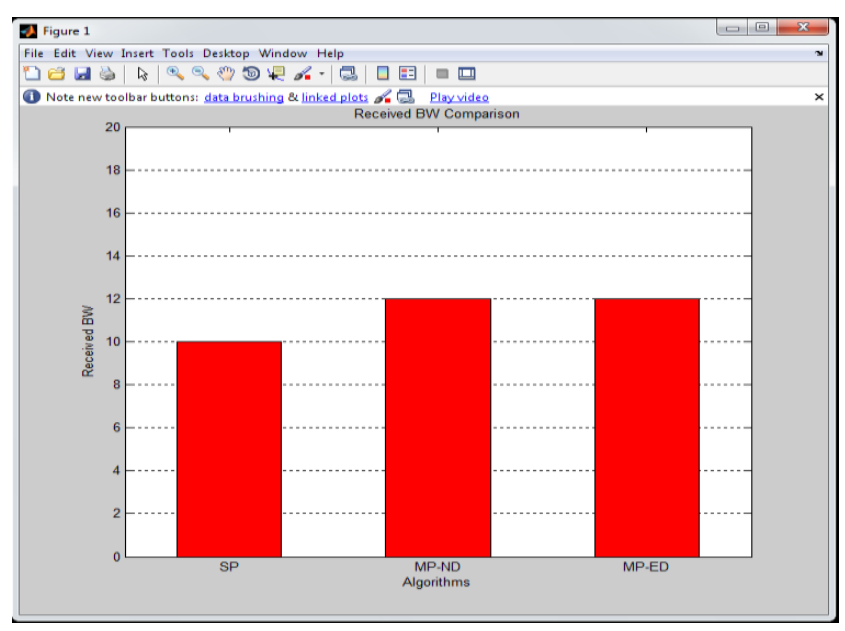

Figure 2: Received Bandwidth.

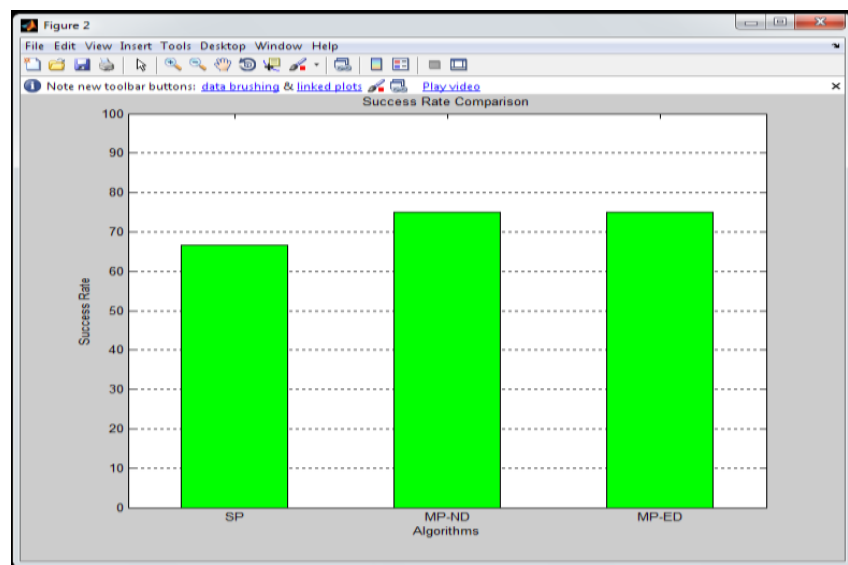

Figure 3: Success Rate

Form experimental data, one can easily observe that bandwidth utilization is more in multipath routing as compared to single path routing. More the bandwidth more data can be transmitted at the same time and hence results in a better video or audio transmission with less amount of buffering. Also the success rate of packets transmission is found to be better in multipath routing as compared to single path routing, which again indicates that multipath routing is better way to transmit data as compared to single path routing.

\section{CONCLUSION}

From this paper it can be concluded that multipath routing is better than single path routing. Paper is basically a case study for comparison of single path routing and multipath routing. For future work we can use these results to improve our network efficiency. We can use better multipath routing techniques than disjoint node and disjoint edge using different parameters/metrics for improving efficiency of networks.

\section{REFERENCES}

[1] E. W. Dijkstra, "“A note on two problems in connexion with graphs"," Numerische Mathematik, vol. vol. 1, pp. 269-271, 1959.

[2] R. Bellman, "“On a routing problem"," Quarterly of Applied Mathematics, vol. vol. 16, pp. 87-90, 1958

[3] S. Nelakuditi and Z.-L. Zhang, "“'On selection of paths for multipath," Proc. IWQoS, pp. 176-186, 2001.

[4] C. Hopps, "“Analysis of an Equal-Cost Multi-Path algorithm"," vol. RFC2992, no. November 2000

[5] Dariusz Bursztynowski, Mateusz Dzida Tomasz Janaszka, "On Popularity-Based Load Balancing in Content," 2012.

[6] Siemens AG, Franz-Josef Götz, Siemens AG Michael Bahr, Multi-path Link-state Routing, January January 162012, IEEE 802.1 AVB TG Meeting, Munich, Germany.

[7] Srihari Nelakuditi and Zhi-Li Zhang, "On Selection of Paths for Multipath Routing," pp. 170-184, 2001.

[8] A. L. Narasimha Reddy Yong Oh Lee, "Disjoint MultiPath Routing and Failure Recovery," pp. 1-12, 2010.

[9] Dariusz Bursztynowski ,Mateusz Dzida Tomasz Janaszka, "On Popularity-Based Load Balancing in Content," pp. 1-8, 2011.

[10] S.Varadarajan2 N.Krishna Chaitanya1, "Load Distribution using Multipath-Routing In Data Networks:A Comparative Study," pp. 1-5, 2015.

[11] Farabi Iqbal and Fernando A. Kuipers, "DISJOINT PATHS IN NETWORKS," pp. 1-14, 2015. 